\title{
Atrial volume and function during exercise in health and disease
}

\author{
Frédéric Schnell ${ }^{1,2^{*}} \mathbb{D}$, Guido Claessen ${ }^{1}$, André La Gerche ${ }^{1,3}$, Piet Claus ${ }^{4}$, Jan Bogaert ${ }^{5}$, Marion Delcroix ${ }^{6}$, \\ François Carré ${ }^{2}$ and Hein Heidbuchel ${ }^{1,7}$
}

\begin{abstract}
Background: Although atrial function has prognostic significance in many cardiovascular conditions, changes during exercise have not previously been assessed. The aim of this study was to evaluate left atrial (LA) and right atrial (RA) volume and function during incremental exercise, both in normal individuals, healthy athletes, and in patients with chronic thromboembolic pulmonary hypertension (CTEPH).
\end{abstract}

Methods: Fifteen healthy non-athletes, 15 athletes and 15 CTEPH patients underwent multi-slice real-time cardiovascular magnetic resonance imaging at rest and during supine bicycle exercise with simultaneous invasive hemodynamic measurements.

Results: At rest, athletes had larger indexed maximal RA and LA volumes (iRAVmax, iLAVmax) than CTEPH patients and non-athletes, the latter two groups having similar values. CTEPH patients had lower RA and LA emptying functions (EmF) at rest. During exercise, RA volumes (maximum and minimum) increased in CTEPH patients, whilst decreasing in athletes and non-athletes $(P<0.001)$. The exercise-induced change in iLAVmax was similar between groups, but iLAVmin did not decrease in CTEPH patients. Thus exercise-induced increases in RAEmF and LAEmF, as seen in normal physiology, were significantly impaired in CTEPH patients. At peak exercise, RA volumes (maximum and minimum) and EmF correlated strongly with RA pressure $(R=0.70 ; P=0.005 ; R=0.83 ; P<0.001 ; R=-0.87 ; P<0.001)$. On multivariate analysis, peak exercise RAEmF and iLAVmin were independent predictors of $\mathrm{VO}_{2}$ peak in $\mathrm{CTEPH}$ patients and together explained $72 \%$ of the variance in $\mathrm{VO}_{2}$ peak ( $B=0.581$ and $B=-0.515$, respectively).

Conclusions: In normal physiology, RAEmF and LAEmF increase with exercise, whereas CTEPH patients have impaired LAEmF and RAEmF, which becomes more apparent during exercise. Therefore, the changes in atrial volumes and function during exercise enable a far better distinction between physiological and pathological atrial remodeling than resting measures of volumes which are prone to confounding factors (e.g. endurance training). Peak exercise RAEmF is a good marker of poor functional state in CTEPH patients.

Keywords: Atrium, Exercise, CTEPH, Athletes, Cardiovascular magnetic resonance

\section{Background}

Although atrial volume and function have prognostic significance in many cardiovascular conditions [1-3], there are scarce data pertaining to changes in atrial volumes during exercise in health $[4,5]$ and none in disease. This is largely due to the limitations of assessing atrial volumes during exercise using transthoracic echocardiography or

\footnotetext{
* Correspondence: frederic.schnell@gmail.com; frederic.schnell@chu-rennes.fr 1 Department of Cardiology, University Hospital Gasthuisberg, University of Leuven, Leuven, Belgium

${ }^{2}$ Department of Sport Medicine, University Hospital Pontchaillou, Rennes 1 University, Rennes, France

Full list of author information is available at the end of the article
}

nuclear imaging techniques. Over the last decades, cardiovascular magnetic resonance (CMR) has emerged as a valid technique to measure atrial volumes at rest [6]. Moreover, development of real-time CMR has enabled the evaluation of cardiac volumes during exercise [7, 8].

In patients with pulmonary hypertension, resting right atrial (RA) volume is a strong predictors of outcome [9-11]. It has been shown that insufficient right ventricular (RV) contractile reserve during exercise is an another important predictor of prognosis among patients with chronic thromboembolic pulmonary hypertension 
(CTEPH) [12-14]. During exercise, the increase in RV contractility in CTPEH patients is insufficient to match the disproportionate increase in afterload. As a consequence, uncoupling occurs between the RV and the pulmonary circulation, which is associated with RV dilation, impaired stroke volume augmentation and a rise in mean RA pressure $[15,16]$. Therefore, exerciseinduced changes in atrial volumes and function during exercise may provide additional information over RV resting measures.

In this study, we sought to evaluate left atrial (LA) and RA volumes and function with CMR during exercise in healthy untrained subjects and in patients with CTEPH. We also included a cohort of healthy endurance athletes in whom atrial function would be expected to be preserved in spite of significant atrial enlargement [17]. We wanted to explore whether atrial function would enable a better differentiation between physiological and pathological cardiac remodeling and correlate better with invasive hemodynamics and exercise capacity than resting measures.

\section{Methods}

\section{Study subjects}

We prospectively included 15 healthy sedentary non-athletes, 15 healthy competitive endurance athletes and 15 CTEPH patients. Diagnosis of CTEPH was established in all patients by ventilation/perfusion scan, pulmonary angiography and right heart catheterization in accordance with contemporary guidelines [18]. None of the patients were receiving pulmonary arterial hypertension specific therapy, all were symptomatic but still able to exercise (NYHA class 2 or 3$)$. We excluded patients with significant abnormal left ventricular (LV) function as a result of coronary artery disease or other cardiomyopathy, or with significant valvular heart disease as assessed previously with an exercise echocardiography.

Athletes were recruited from advertisements at local triathlon and cycling clubs and were included if they were participating in regular cycling and/or running training of $>6 \mathrm{~h} /$ week. Non-athletes were recruited from advertisements among hospital staff members and were included if they were not engaged in regular sport's practice (i.e. $\leq 1 \mathrm{~h} /$ week). None of the athletes and non-athletes met the exclusion criteria of known cardiovascular disease, symptoms, risk factors or abnormalities on electrocardiography and exercise transthoracic echocardiogram.

The study protocol conformed to the Declaration of Helsinki and was approved by the ethics committee of UZ Leuven ( $\mathrm{N}^{\circ}$ B322201214035). All subjects provided informed consent.

\section{Study protocol}

\section{Exercise protocol}

First, cardiopulmonary exercise testing (CPET) was performed on an upright cycle ergometer (ER900 and Oxycon Alpha, Jaeger, Germany) using a continuous ramp protocol until exhaustion. Breath-by-breath analysis provided measures of oxygen consumption at peak exercise $\left(\mathrm{VO}_{2}\right.$ peak) and maximal power output in Watts (Pmax).

Twenty-four hours later, all subjects underwent exercise CMR with simultaneous invasive pressure measurement. Prior to exercise, a 7 Fr pulmonary artery catheter was inserted in the internal jugular vein and guided under fluoroscopy or pressure curve monitoring to the proximal right main pulmonary artery. A 20 gauge arterial catheter was placed in the radial artery. In the CMR suite, these catheters were attached to CMRcompatible pressure transducers that were connected to a PowerLab recording system (AD Instruments, Oxford, United Kingdom).

Patients underwent CMR at rest and then during exercise at 25\%, 50\% and 66\% of Pmax determined during CPET. We have previously demonstrated that $66 \%$ of the maximal upright exercise power (in Watts) corresponded to the maximal sustainable exercise intensity in a supine position, i.e. peak-intensity exercise [8]. During the CMR protocol, pulmonary and systemic arterial pressures were continuously recorded by the pulmonary and radial artery catheters and analyzed offline using LabChart v6.1.1 (AD Instruments). All pressure measurements were averaged over 10 consecutive cardiac cycles during unrestricted respiration [19]. Due to technical considerations, mean RA pressure was only recorded in a subgroup of non-athletes $(n=6)$ and CTEPH patients $(n=10)$.

\section{CMR equipment, image acquisition, and analysis}

Cardiac volumes were measured during supine cycling exercise using a real-time CMR method that we previously described in detail and have validated against invasive standards [8]. In brief: subjects performed supine exercise within the CMR bore using a cycle ergometer with adjustable electronic resistance (Lode, Groningen, The Netherlands). Images were acquired with a $1.5 \mathrm{~T}$ CMR scanner (Philips Achieva, Philips Healthcare, Best, The Netherlands) with a five-element phased-array coil.

Balanced steady-state free-precession cine imaging was performed without cardiac gating. Imaging parameters were as follows: field of view, $320 \times 260 \mathrm{~mm}$ (approximately); matrix, $128 \times 128$; flip angle, $50^{\circ}$; SENSE factor, 2 (Cartesian k-space undersampling); repetition time, $1.8 \mathrm{~ms}$; echo time, $0.9 \mathrm{~ms}$; and reconstructed voxel size, $2.3 \times 2.3 \times 8 \mathrm{~mm}$. A three-dimensional (3D) stack of 13-18 
contiguous $8 \mathrm{~mm}$ images slices covering the whole heart from the apex to the base was serially acquired in the short-axis plane and subsequently in the horizontal long-axis plane. At rest, each slice level consisted of 100 consecutive image frames in the short axis and in the horizontal long axis plane. All images frames were acquired during free breathing with a temporal resolution of $36-38 \mathrm{~ms}$. Therefore, according to heart rate the average number of images per cardiac cycle was approximately 22 to 27 at rest and 10 to 13 at peak exercise. For the exercise CMR protocol, a reduced number of repetitions (from 100 frames at rest to 60 frames/slice at maximal-intensity exercise) were programmed for each increase in exercise intensity, but there was sufficient time to acquire numerous cardiac cycles and at least one complete respiratory cycle at each slice of the cine acquisition. In order to obtain a stable heart rate during the acquisition of the whole myocardial volume at each exercise level, the acquisitions were started after a few minutes of exercise, in a relative steady state situation.

Simultaneous with the image acquisition, information on the timing of respiration was obtained by measuring abdominal pressure with a plethysmograph placed on the upper abdomen, and electrocardiographic R-wave determination was derived from a hemodynamic monitor (Maglife Serenity, Schiller, Baar, Switzerland). These physiological data were retrospectively synchronized with the images using an in-house developed software program (RightVol, Leuven, Belgium) such that contouring could be performed at the same point of the respiratory cycle for all slices to minimize through-plane motion [8].

LV and RV endocardial contours were manually traced on a stack of short-axis image slices with simultaneous reference to the horizontal long-axis plane thus enabling the analyzer to confirm the position of the atrioventricular plane. Similarly, LA and RA endocardial contours were manually traced on a stack of horizontal long-axis image slices with simultaneous reference to the short-axis (Fig. 1). The ability of the software to contour simultaneously two orthogonal planes enabled to crosscheck contouring of the right structures and to smooth the effect of a slight change in heart rate during the acquisition. LA and RA maximal (iLAVmax, iRAVmax) and minimal (iLAVmin, iRAVmin) volumes and biventricular end-diastolic and end-systolic volumes (EDV and ESV) were calculated using a summation of disks technique. Volumes were indexed for body surface area. LV and RV stroke volume were measured as the difference between EDV and ESV. Cardiac output was measured as stroke volume $\mathrm{x}$ heart rate, whilst left ventricular and right ventriuclar ejection fraction (LVEF, RVEF) was calculated as stroke volume/EDV. Atrial stroke volume was calculated as [maximal - minimal atrial volume] [2]. As a measure of atrial function, left atrial and right atrial emptying function (LAEmF, RAEmF) was calculated as [(atrial maximal volume -
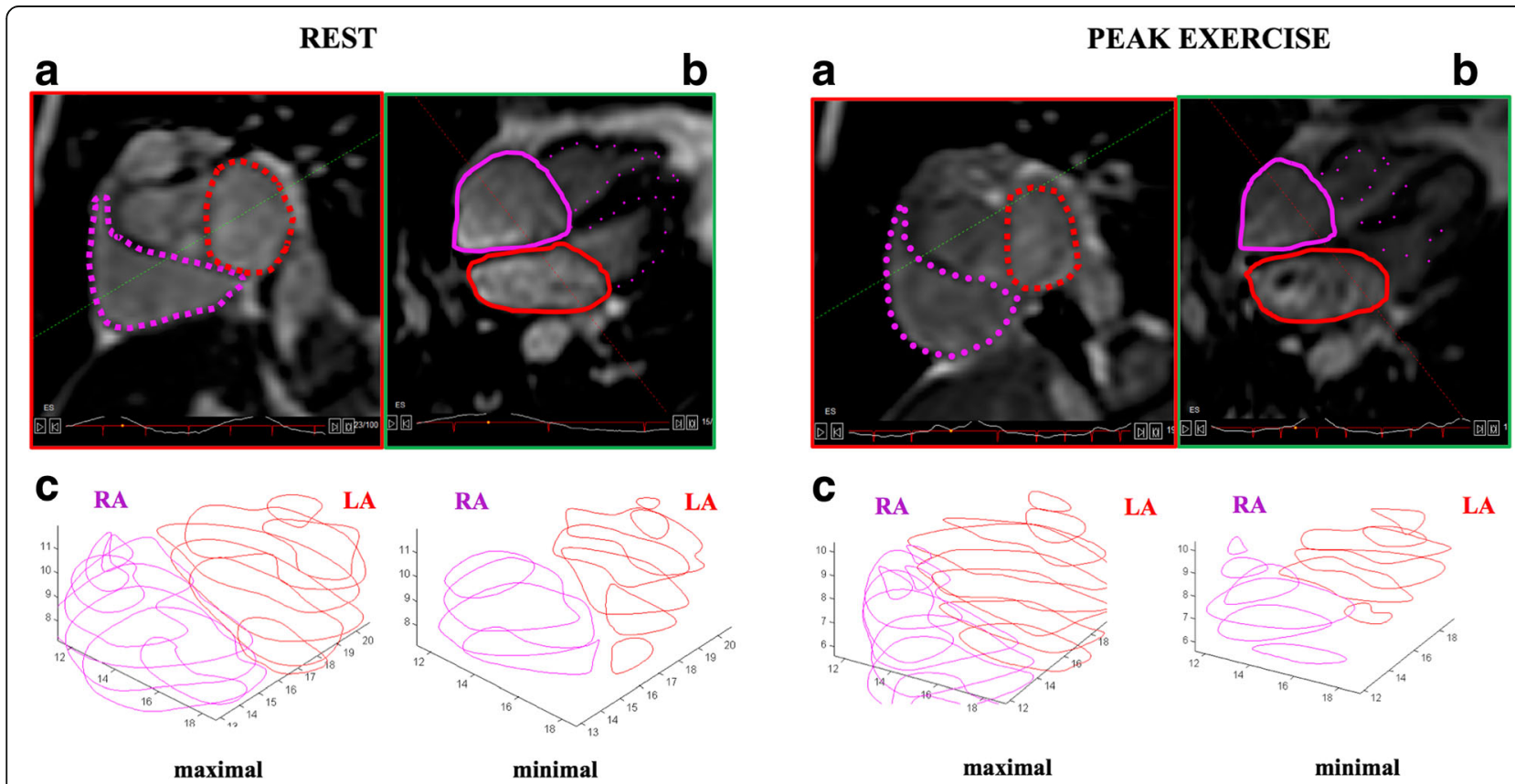

LA

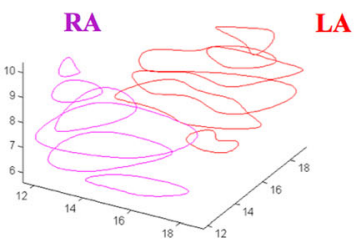

maximal

minimal

Fig. 1 Example of an atrial contouring at rest and at peak exercise. Panel a: short axis view (SAX) at atrial level; panel b: horizontal long axis view (HLA); green dotted line: intersection of HLA on SAX; red dotted line: intersection of SAX on HLA. Panel c: right atrial (RA) and left atrial (LA) maximal and minimal volumes 
atrial minimal volume)/atrial maximal volume] [3, 6], whilst atrial reservoir function was calculated as [(atrial maximal volume - atrial minimal volume)/atrial minimal volume] [20].

\section{Statistical analysis}

Gaussian distribution of all continuous variables was confirmed with a Kolmogorov-Smirnov test, and values are reported as mean $\pm \mathrm{SD}$. A 2-tailed value of $P<0.05$ was considered significant. Differences between baseline and peak exercise measures were analyzed with a paired $t$ test, whereas comparisons between groups during exercise were performed with repeated measures ANOVA. Correlations were quantified by simple linear regression, a multivariate linear regression was applied to identify the independent predictors of $\mathrm{VO}_{2}$ peak including all covariates that were significant in the univariable analyses at the $\mathrm{P}<0.05$ level, we used a backward elimination procedure with the other variables. To study the confounding effect of age, a subgroup analysis was performed on 5 patients of similar age from each group. Intra- and inter-observer variability of atrial volumes were expressed by intraclass correlation coefficients in 5 subjects randomly selected in each group (2 CETPH, 2 athletes, 1 non-athlete), (an additional figure shows this more in details: Additional file 1). Statistical analysis was performed using SPSS (version 16.0, International Business Machines, Armonk, New York, USA).

\section{Results}

\section{Baseline characteristics}

Demographic characteristics are detailed in Table 1 . CTEPH patients were older than non-athletes and athletes, whilst there was no difference in gender. $\mathrm{VO}_{2}$ peak was significantly lower in CTEPH patients than in healthy subjects, and lower in non-athletes than in athletes $(13.8 \pm 3.0$ vs. $35.0 \pm 7.1$ vs. $55.3 \pm 10.5 \mathrm{ml} / \mathrm{min} / \mathrm{kg}$, respectively; $P<0.001$ ).

\section{Atrial volumes and function at rest and during exercise}

At rest, athletes had larger iRAVmax and iLAVmax than CTEPH patients and non-athletes, the latter two groups having similar values (Table 2, Fig. 2). Resting iLAVmin was higher in athletes than in non-athletes and CTEPH patients. iRAVmin was larger in athletes compared to non-athletes, but similar to CTEPH patients. At rest, both RAEmF and LAEmF were lower in CTEPH compared to athletes and non-athletes.

From rest to peak exercise, iRAVmax and iRAVmin increased in CTEPH patients, whilst decreasing in athletes and non-athletes $(\mathrm{P}<0.001$ for interaction between exercise-intensity and group). Whilst the exercise-induced change in iLAVmax was similar between groups, iLAVmin decreased in non-athletes and athletes but not in CTEPH patients $(P<0.05$ for interaction). As a result, exerciseinduced increases in RAEmF and LAEmF as well as LA and RA reservoir were significantly impaired in CTEPH patients. Moreover, atrial remodeling was not symmetrical in CTEPH with a more dilated RA in comparison with LA or RV, as shown by a more elevated (RA/LA) maximal ratio and (RA/RV) diastolic ratio in CTEPH (Table 2).

\section{Subgroup analysis: Effect of age on atrial volumes and function}

Analysis was performed on 5 patients from each group with similar ages (mean age of $52.4 \pm 7.1 ; 45.0 \pm 13.3$ and $43.2 \pm$ 5.8 years in non-athletes, CTEPH patients and athletes respectively; $P=0.29$ ) (an additional table shows this more in details: Additional file 2). Despite smaller group size, exercise changes in RA volumes remained significant with the same pattern of augmented function during exercise in healthy subjects and RA dysfunction in the CTEPH patients. The magnitude of these opposing effects was similar to that of the global population from which the agematched sub-groups were derived (Fig. 3, an additional table shows this more in details: Additional file 3).

Table 1 Patients characteristics

\begin{tabular}{|c|c|c|c|c|}
\hline Parameter & $\begin{array}{l}\text { Non-Athletes } \\
(n=15)\end{array}$ & $\begin{array}{l}\text { CTEPH } \\
(n=15)\end{array}$ & $\begin{array}{l}\text { Athletes } \\
(n=15)\end{array}$ & $P$-value \\
\hline \multicolumn{5}{|l|}{ Demographics } \\
\hline Gender (female) & 3 & 5 & 0 & 0.056 \\
\hline Age (years) & $35.1 \pm 14.2$ & $60.9 \pm 15.6 \dagger$ & $34.6 \pm 7.8^{*}$ & $<0.001$ \\
\hline $\mathrm{BSA}\left(\mathrm{m}^{2}\right)$ & $1.9 \pm 0.2$ & $1.9 \pm 0.3$ & $2.0 \pm 0.1$ & 0.531 \\
\hline \multicolumn{5}{|l|}{ CPET } \\
\hline Maximal HR (bpm) & $172.0 \pm 18.0$ & $131.0 \pm 22.9+$ & $180.1 \pm 10.4^{*}$ & $<0.001$ \\
\hline Maximal power (Watts) & $221.8 \pm 68.2$ & $83.2 \pm 29.5+$ & $376.0 \pm 65.0^{*} \neq$ & $<0.001$ \\
\hline $\mathrm{VO}_{2}$ peak $(\mathrm{ml} / \mathrm{min} / \mathrm{kg})$ & $35.0 \pm 7.1$ & $13.8 \pm 3.0 \dagger$ & $55.3 \pm 10.5^{*} \neq$ & $<0.001$ \\
\hline
\end{tabular}

$B S A$ body surface area, CPET cardiopulmonary exercise test, $H R$ heart rate, VO2 peak peak oxygen consumption

${ }^{*}$ Athletes vs. CTEPH; † CTEPH vs. Non-Athletes; ¥Athletes vs. Non-Athletes 
Table 2 CMR volumes and hemodynamics at rest and peak exercise

\begin{tabular}{|c|c|c|c|c|c|}
\hline & & $\begin{array}{l}\text { Non-Athletes } \\
(n=15)\end{array}$ & $\begin{array}{l}\text { CTEPH } \\
(n=15)\end{array}$ & $\begin{array}{l}\text { Athletes } \\
(n=15)\end{array}$ & $P$-value \\
\hline LVEDVi $\left(\mathrm{ml} / \mathrm{m}^{2}\right)$ & $\begin{array}{l}\text { Rest } \\
\text { Peak Ex }\end{array}$ & $\begin{array}{l}87.9 \pm 16.0 \\
84.1 \pm 15.1\end{array}$ & $\begin{array}{l}58.1 \pm 11.7 \dagger \\
51.9 \pm 14.8 \dagger\end{array}$ & $\begin{array}{l}118.0 \pm 12.7^{*} \neq \\
116.4 \pm 13.7^{*} \neq\end{array}$ & $\begin{array}{l}<0.001 \\
<0.001\end{array}$ \\
\hline LVESVi $\left(\mathrm{ml} / \mathrm{m}^{2}\right)$ & $\begin{array}{l}\text { Rest } \\
\text { Peak Ex }\end{array}$ & $\begin{array}{l}36.0 \pm 9.0 \\
26.3 \pm 5.0\end{array}$ & $\begin{array}{l}23.5 \pm 8.3 \dagger \\
18.7 \pm 9.8 \dagger\end{array}$ & $\begin{array}{l}47.9 \pm 9.8^{*} \neq \\
35.8 \pm 8.1^{*} \neq\end{array}$ & $\begin{array}{l}<0.001 \\
<0.001\end{array}$ \\
\hline LVSVi & $\begin{array}{l}\text { Rest } \\
\text { Peak Ex }\end{array}$ & $\begin{array}{l}51.9 \pm 10.1 \\
57.8 \pm 11.8\end{array}$ & $\begin{array}{l}34.6 \pm 7.2 \dagger \\
33.3 \pm 10.1 \dagger\end{array}$ & $\begin{array}{l}70.1 \pm 7.6^{*} \neq \\
80.6 \pm 10.1^{*} \neq\end{array}$ & $\begin{array}{l}<0.001 \\
<0.001\end{array}$ \\
\hline LVEF (\%) & $\begin{array}{l}\text { Rest } \\
\text { Peak Ex }\end{array}$ & $\begin{array}{l}59.3 \pm 5.7 \\
68.6 \pm 4.2\end{array}$ & $\begin{array}{l}60.2 \pm 9.3 \\
65.2 \pm 12.6\end{array}$ & $\begin{array}{l}59.6 \pm 5.2 \\
69.3 \pm 5.4\end{array}$ & $\begin{array}{l}0.939 \\
0.353\end{array}$ \\
\hline RVEDVi $\left(\mathrm{ml} / \mathrm{m}^{2}\right)$ & $\begin{array}{l}\text { Rest } \\
\text { Peak Ex }\end{array}$ & $\begin{array}{l}87.8 \pm 18.3 \\
80.6 \pm 15.1\end{array}$ & $\begin{array}{l}89.3 \pm 17.3 \\
100.9 \pm 14.1+\end{array}$ & $\begin{array}{l}122.8 \pm 20.0^{*} \neq \\
117.2 \pm 24.5 \neq\end{array}$ & $\begin{array}{l}<0.001 \\
<0.001\end{array}$ \\
\hline RVESVi $\left(\mathrm{ml} / \mathrm{m}^{2}\right)$ & $\begin{array}{l}\text { Rest } \\
\text { Peak Ex }\end{array}$ & $\begin{array}{l}37.2 \pm 10.0 \\
23.3 \pm 6.1\end{array}$ & $\begin{array}{l}57.7 \pm 14.8 \dagger \\
66.3 \pm 14.5 \dagger\end{array}$ & $\begin{array}{l}52.9 \pm 11.8 \neq \\
38.6 \pm 15.3^{*} \neq\end{array}$ & $\begin{array}{l}<0.001 \\
<0.001\end{array}$ \\
\hline RVSVi & $\begin{array}{l}\text { Rest } \\
\text { Peak Ex }\end{array}$ & $\begin{array}{l}50.6 \pm 9.8 \\
57.3 \pm 11.0\end{array}$ & $\begin{array}{l}31.6 \pm 6.4+ \\
34.6 \pm 8.5+\end{array}$ & $\begin{array}{l}69.9 \pm 9.9^{*} \neq \\
78.6 \pm 12.3^{*} \neq\end{array}$ & $\begin{array}{l}<0.001 \\
<0.001\end{array}$ \\
\hline RVEF (\%) & $\begin{array}{l}\text { Rest } \\
\text { Peak Ex }\end{array}$ & $\begin{array}{l}57.9 \pm 4.5 \\
71.2 \pm 5.0\end{array}$ & $\begin{array}{l}35.8 \pm 6.3 \dagger \\
34.7 \pm 8.5 \dagger\end{array}$ & $\begin{array}{l}57.2 \pm 3.8^{*} \\
67.9 \pm 6.8^{*}\end{array}$ & $\begin{array}{l}<0.001 \\
<0.001\end{array}$ \\
\hline Cardiac Index $\left(1 / \mathrm{min} / \mathrm{m}^{2}\right)$ & $\begin{array}{l}\text { Rest } \\
\text { Peak Ex }\end{array}$ & $\begin{array}{l}3.4 \pm 0.8 \\
8.7 \pm 2.1\end{array}$ & $\begin{array}{l}2.6 \pm 0.7 \dagger \\
4.1 \pm 1.0 \dagger\end{array}$ & $\begin{array}{l}4.1 \pm 1.1^{*} \\
12.3 \pm 2.3^{*} \neq\end{array}$ & $\begin{array}{l}<0.001 \\
<0.001\end{array}$ \\
\hline iLAVmax $\left(\mathrm{ml} / \mathrm{m}^{2}\right)$ & $\begin{array}{l}\text { Rest } \\
\text { Peak Ex }\end{array}$ & $\begin{array}{l}39.5 \pm 10.1 \\
38.3 \pm 11.4\end{array}$ & $\begin{array}{l}30.9 \pm 9.1 \\
26.3 \pm 7.9+\end{array}$ & $\begin{array}{l}55.8 \pm 9.2^{*} \neq \\
53.5 \pm 9.7^{*} \neq\end{array}$ & $\begin{array}{l}<0.001 \\
<0.001\end{array}$ \\
\hline $\mathrm{iLAV \operatorname {min }}\left(\mathrm{ml} / \mathrm{m}^{2}\right)$ & $\begin{array}{l}\text { Rest } \\
\text { Peak Ex }\end{array}$ & $\begin{array}{l}17.7 \pm 5.3 \\
13.3 \pm 5.6\end{array}$ & $\begin{array}{l}17.2 \pm 6.4 \\
15.8 \pm 6.7\end{array}$ & $\begin{array}{l}25.6 \pm 5.0^{*} \neq \\
20.2 \pm 6.4 \neq\end{array}$ & $\begin{array}{l}<0.001 \\
0.023\end{array}$ \\
\hline LA Stroke Volume index & $\begin{array}{l}\text { Rest } \\
\text { Peak Ex }\end{array}$ & $\begin{array}{l}21.8 \pm 6.4 \\
24.9 \pm 6.6\end{array}$ & $\begin{array}{l}13.7 \pm 4.9+ \\
10.5 \pm 6.6 \dagger\end{array}$ & $\begin{array}{l}30.1 \pm 5.7^{*} \neq \\
33.3 \pm 8.1^{*} \neq\end{array}$ & $\begin{array}{l}<0.001 \\
<0.001\end{array}$ \\
\hline LAEmF (\%) & $\begin{array}{l}\text { Rest } \\
\text { Peak Ex }\end{array}$ & $\begin{array}{l}54.9 \pm 7.8 \\
66.1 \pm 6.4\end{array}$ & $\begin{array}{l}44.6 \pm 11.4 \dagger \\
39.2 \pm 20.7 \dagger\end{array}$ & $\begin{array}{l}54.0 \pm 5.1^{*} \\
62.1 \pm 10.6^{*}\end{array}$ & $\begin{array}{l}0.003 \\
<0.001\end{array}$ \\
\hline LA reservoir & $\begin{array}{l}\text { Rest } \\
\text { Peak Ex }\end{array}$ & $\begin{array}{l}1.29 \pm 0.5 \\
2.05 \pm 0.60\end{array}$ & $\begin{array}{l}0.88 \pm 0.43 \dagger \\
0.88 \pm 0.80 \dagger\end{array}$ & $\begin{array}{l}1.20 \pm 0.24 \\
1.83 \pm 0.80^{*}\end{array}$ & $\begin{array}{l}0.020 \\
<0.001\end{array}$ \\
\hline iRAVmax $\left(\mathrm{ml} / \mathrm{m}^{2}\right)$ & $\begin{array}{l}\text { Rest } \\
\text { Peak Ex }\end{array}$ & $\begin{array}{l}53.4 \pm 11.3 \\
44.7 \pm 10.7\end{array}$ & $\begin{array}{l}63.6 \pm 18.9 \\
78.8 \pm 25.8+\end{array}$ & $\begin{array}{l}79.9 \pm 19.2^{*} \neq \\
72.0 \pm 21.4 \neq\end{array}$ & $\begin{array}{l}<0.001 \\
<0.001\end{array}$ \\
\hline iRAVmin $\left(\mathrm{ml} / \mathrm{m}^{2}\right)$ & $\begin{array}{l}\text { Rest } \\
\text { Peak Ex }\end{array}$ & $\begin{array}{l}26.4 \pm 6.5 \\
16.1 \pm 2.9\end{array}$ & $\begin{array}{l}37.8 \pm 16.7 \\
54.7 \pm 24.9+\end{array}$ & $\begin{array}{l}39.0 \pm 12.2 \neq \\
26.8 \pm 13.7^{*}\end{array}$ & $\begin{array}{l}0.016 \\
<0.001\end{array}$ \\
\hline RA Stroke Volume Index & $\begin{array}{l}\text { Rest } \\
\text { Peak Ex }\end{array}$ & $\begin{array}{l}26.9 \pm 7.9 \\
28.5 \pm 8.8\end{array}$ & $\begin{array}{l}25.8 \pm 5.5 \\
24.2 \pm 8.1\end{array}$ & $\begin{array}{l}40.9 \pm 9.2^{*} \neq \\
45.1 \pm 12.3\end{array}$ & $\begin{array}{l}<0.001 \\
<0.001\end{array}$ \\
\hline RAEmF (\%) & $\begin{array}{l}\text { Rest } \\
\text { Peak Ex }\end{array}$ & $\begin{array}{l}50.1 \pm 8.9 \\
63.0 \pm 6.4\end{array}$ & $\begin{array}{l}42.6 \pm 10.9 \\
32.8 \pm 12.8 \dagger\end{array}$ & $\begin{array}{l}51.7 \pm 6.3^{*} \\
63.5 \pm 11.2^{*}\end{array}$ & $\begin{array}{l}0.017 \\
<0.001\end{array}$ \\
\hline RA reservoir & $\begin{array}{l}\text { Rest } \\
\text { Peak Ex }\end{array}$ & $\begin{array}{l}1.07 \pm 0.39 \\
1.78 \pm 0.46\end{array}$ & $\begin{array}{l}0.80 \pm 0.35 \\
0.54 \pm 0.30 \dagger\end{array}$ & $\begin{array}{l}1.11 \pm 0.31 \\
2.02 \pm 1.04^{*}\end{array}$ & $\begin{array}{l}0.046 \\
<0.001\end{array}$ \\
\hline (RA Volume/LA volume) max & $\begin{array}{l}\text { Rest } \\
\text { Peak Ex }\end{array}$ & $\begin{array}{l}1.38 \pm 0.20 \\
1.20 \pm 0.19\end{array}$ & $\begin{array}{l}2.20 \pm 0.86 \dagger \\
3.21 \pm 1.36 \dagger\end{array}$ & $\begin{array}{l}1.44 \pm 0.30^{*} \\
1.35 \pm 0.32^{*}\end{array}$ & $\begin{array}{l}<0.001 \\
<0.001\end{array}$ \\
\hline RAVmin/RVEDV & $\begin{array}{l}\text { Rest } \\
\text { Peak Ex }\end{array}$ & $\begin{array}{l}0.31 \pm 0.06 \\
0.20 \pm 0.04\end{array}$ & $\begin{array}{l}0.42 \pm 0.17 \dagger \\
0.53 \pm 0.23 \dagger\end{array}$ & $\begin{array}{l}0.32 \pm 0.07^{*} \\
0.23 \pm 0.09^{*}\end{array}$ & $\begin{array}{l}0.011 \\
<0.001\end{array}$ \\
\hline $\mathrm{HR}(\mathrm{bpm})$ & $\begin{array}{l}\text { Rest } \\
\text { Peak Ex }\end{array}$ & $\begin{array}{l}66.4 \pm 6.7 \\
149.6 \pm 11.7\end{array}$ & $\begin{array}{l}76.9 \pm 12.4 \dagger \\
124.2 \pm 22.1+\end{array}$ & $\begin{array}{l}58.7 \pm 11.9^{*} \\
154.6 \pm 17.8^{*}\end{array}$ & $\begin{array}{l}<0.001 \\
<0.001\end{array}$ \\
\hline Systolic BP (mmHg) & $\begin{array}{l}\text { Rest } \\
\text { Peak Ex }\end{array}$ & $\begin{array}{l}137.0 \pm 20.5 \\
183.6 \pm 31.5\end{array}$ & $\begin{array}{l}137.2 \pm 19.3 \\
175.9 \pm 36.9\end{array}$ & $\begin{array}{l}140.6 \pm 14.2 \\
206.1 \pm 31.6\end{array}$ & $\begin{array}{l}0.834 \\
0.057\end{array}$ \\
\hline Diastlic BP $(\mathrm{mmHg})$ & $\begin{array}{l}\text { Rest } \\
\text { Peak Ex }\end{array}$ & $\begin{array}{l}69.2 \pm 10.4 \\
77.5 \pm 9.8\end{array}$ & $\begin{array}{l}70.40 \pm 12.4 \\
85.57 \pm 16.9\end{array}$ & $\begin{array}{l}69.9 \pm 6.9 \\
78.9 \pm 10.5\end{array}$ & $\begin{array}{l}0.952 \\
0.251\end{array}$ \\
\hline Mean PA pressure $(\mathrm{mmHg})$ & $\begin{array}{l}\text { Rest } \\
\text { Peak Ex }\end{array}$ & $\begin{array}{l}9.6 \pm 2.7 \\
20.6 \pm 6.5\end{array}$ & $\begin{array}{l}43.2 \pm 10.1 \dagger \\
65.9 \pm 11.0 \dagger\end{array}$ & $\begin{array}{l}13.8 \pm 4.0^{*} \\
27.9 \pm 8.0^{*}\end{array}$ & $\begin{array}{l}<0.001 \\
<0.001\end{array}$ \\
\hline Mean RA Pressure $(\mathrm{mmHg})$ & $\begin{array}{l}\text { Rest } \\
\text { Peak Ex }\end{array}$ & $\begin{array}{l}3 \pm 2(n=6) \\
5 \pm 2+(n=6)\end{array}$ & $\begin{array}{l}7 \pm 4(n=10) \\
17 \pm 7(n=9)\end{array}$ & - & $\begin{array}{l}0.058 \\
0.002\end{array}$ \\
\hline
\end{tabular}

Peak Ex peak exercise, LVEDVi, RVEDVi left and right ventricular end diastolic volume index, LVESVi vol., LVSVi, RVSVi left and right ventricular stroke volume index, LASVI, RASVi left and right atrial stroke volume index, LVEF, RVEF left and right ventricular ejection fraction, iLAVmax, iRAVmax indexed left and right maximal volume, iLAVmin, iRAVmin indexed left and right minimal volume, LAEmF, RAEmF left and right atrial emptying function, LA and RA reservoir $L A$ and RA reservoir function, $B P$ blood pressure, $P A$ pulmonary artery

*Athletes vs. CTEPH; † CTEPH vs. Non-Athletes; ¥Athletes vs. Non-Athletes 

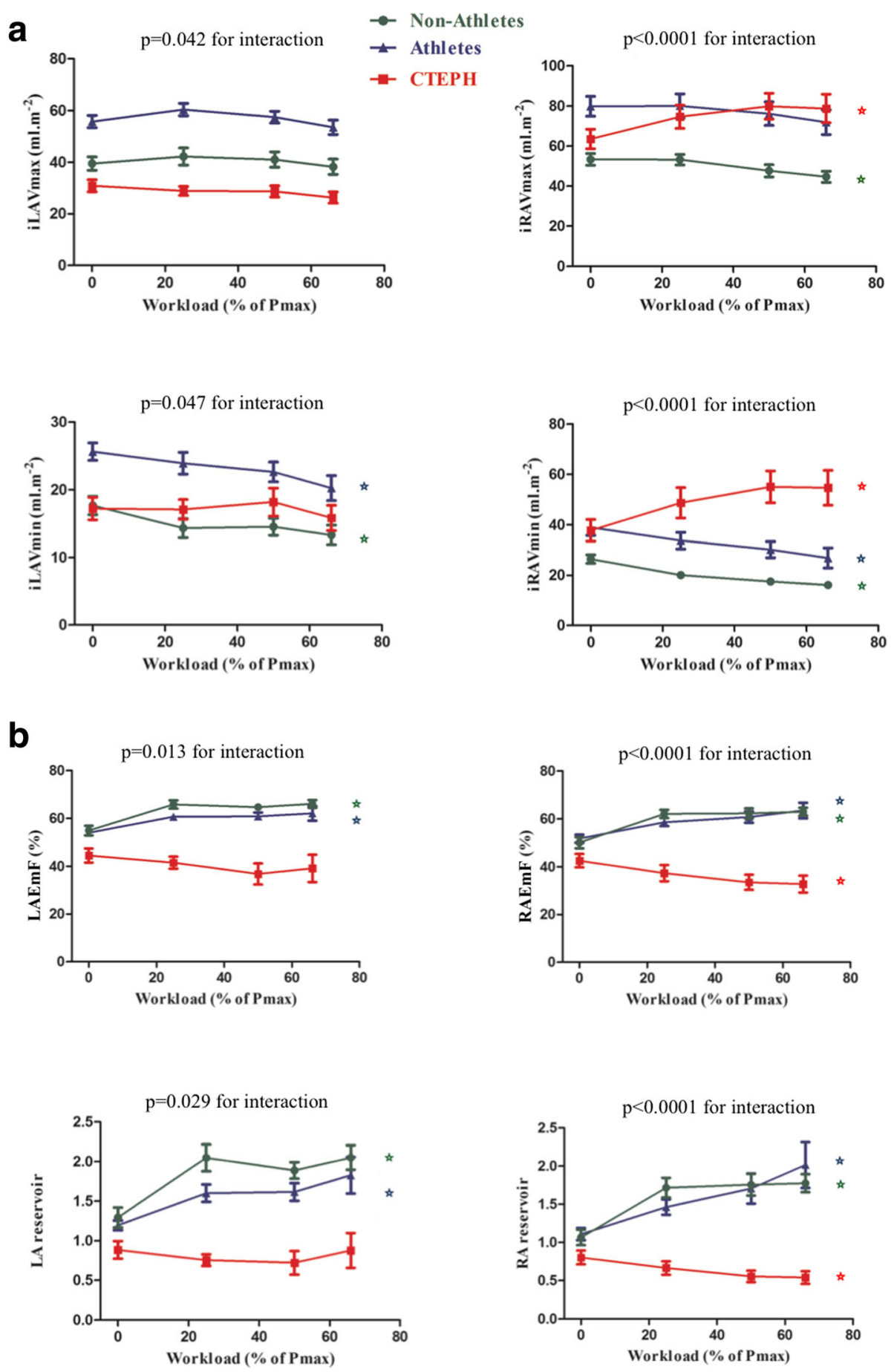

Fig. 2 Atrial volumes and function at rest and exercise. Panel a: Atrial volumes. iLAVmax: indexed left atrial maximal volume; iLAVmin: indexed left atrial minimal volume; iRAV $V_{\text {max }}$ : indexed right atrial maximal volume; iRAVmax: indexed right atrial minimal volume. Panel b: Atrial emptying function. LAEmF: left atrial emptying function; RAEmF: right atrial emptying function; Atrial reservoir was measured as (maximal atrial volume _ minimal atrial volume)/minimal atrial volume. ${ }^{*} P<0.05$ for comparison between peak exercise and rest value

Ventricular function, valvular function and hemodynamics at rest and during exercise

Similar to RA functional changes during exercise, RVEF augmentation was significantly impaired in the CTEPH cohort versus non-athletes and athletes $(-1.1 \pm 6.1 \%, P=$ 0.60 vs. $+13.3 \pm 6.2 \%, P<0.001$ and $+10.7 \pm 4.8 \%$ respectively, $\mathrm{P}<0.001)$. In contrast, the exercise-induced increase in LVEF was similar between the different 

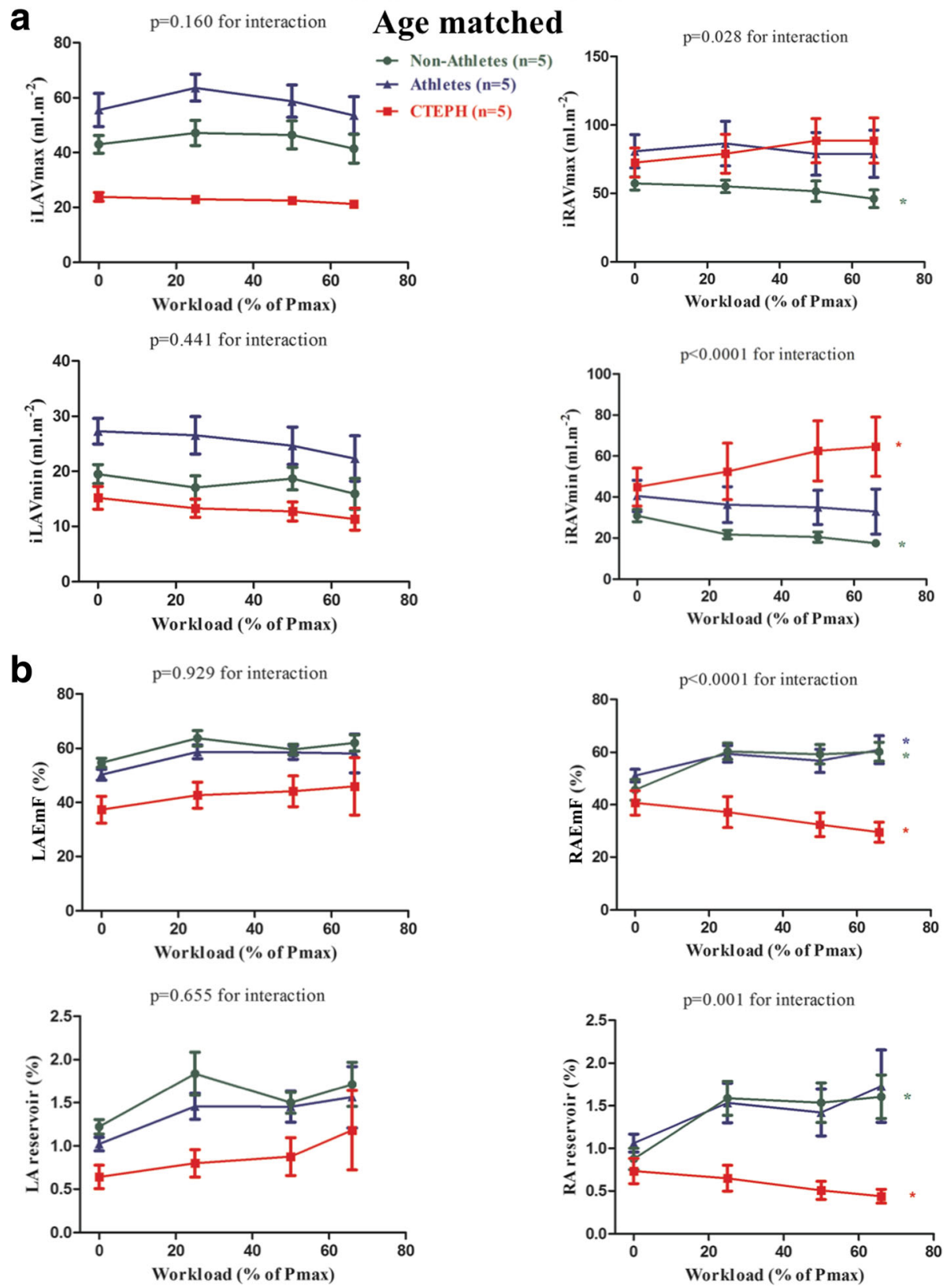

Fig. 3 Atrial volumes and function at rest and exercise in subgroups matched for age. Panel a: Atrial volumes. iLAVmax: indexed left atrial maximal volume; iLAVmin: indexed left atrial minimal volume; $\mathrm{RAV}_{\text {max: }}$ indexed right atrial maximal volume; iRAVmax: indexed right atrial minimal volume. Panel $\mathbf{b}$ : Atrial function. LAEmF: left atrial emptying function; RAEmF: right atrial emptying function; Atrial reservoir was measured as (maximal atrial volume _ minimal atrial volume)/minimal atrial volume. ${ }^{*} \mathrm{P}<0.05$ for comparison between peak exercise and rest value

groups $(+5.4 \pm 7.6 \%, P=0.020$ vs. $+9.3 \pm 6.4 \%, P<0.001$ vs. $+9.7 \pm 5.3 \%, \mathrm{P}<0.001$ in $\mathrm{CTEPH}$, non-athletes and athletes respectively). RVEF correlated with RAEmF $(R=0.55$ and $R=0.84$, respectively for rest and peak exercise; $\mathrm{P}<0.001)$ but also with LAEmF $(\mathrm{R}=0.55$ and $R=0.74$, respectively; $\mathrm{P}<0.001$ ), no correlation was found between LAEmF and LVEF.

None of the CTEPH patients had significant tricuspid regurgitation, as confirmed by only a minimal difference in $\mathrm{LV}$ and RV stroke volume index at rest ( $35 \pm 7$ vs. 32 $\pm 6 \mathrm{ml} / \mathrm{m}^{2}$, respectively; $\left.P=0.004\right)$ and a similar $\mathrm{LV}$ and RV stroke volume index at peak exercise ( $33 \pm 10$ vs. 35 $\pm 8 \mathrm{ml} / \mathrm{m}^{2} ; P=0.22$ ). It has to be mentioned that there was no concordance between ventricular and atrial stroke volume index, neither between LA and RA stroke volume index (Table 2).

At rest, CI was lower in CTEPH patients than in healthy subjects but similar in non-athletes and athletes 
$\left(2.6 \pm 0.7 \mathrm{l} / \mathrm{min} / \mathrm{m}^{2}\right.$ vs. $3.4 \pm 0.8$ vs. $\left.4.1 \pm 1.1 ; P<0.001\right)$. As expected, mean pulmonary pressure was significantly elevated in the CTEPH patients, whilst non-athletes and athletes had normal mean pulmonary artery pressure. During exercise, there was greater mean RA pressure increase relative to cardiac output in CTEPH patients as compared with non-athletes $(P<0.001$; Fig. 4$)$. The peak exercise mean RA pressure was also significantly higher in CTEPH patients than in non-athletes $(17 \pm 7$ vs. $5 \pm$ $2 \mathrm{mmHg}$, respectively; $P=0.001$ ).

At rest, mean RA pressure correlated with RA volumes (iRAVmax: $R=0.72, P=0.002$; iRAVmin: $R=0.77, P<$ 0.001 ) and RA function (RAEmF and RA reservoir: $R=$ -0.58 , both $P=0.018$ ). At peak exercise, the association between mean RA pressure and RA volumes (iRAVmax: $R=0.70 ; P=0.005$; iRAVmin: $R=0.83 ; P<0.001)$ and RA function (RAEmF: $R=-0.87 ; P<0.001$; RA reservoir: $R$ $=-0.77 ; P<0.001)$ remained strong $($ Fig. 4$)$.

\section{Predictors of $\mathrm{VO}_{2}$ peak in the CTEPH group}

Within the group of CTEPH patients, none of the resting measurements correlated with $\mathrm{VO}_{2}$ peak on univariate analysis. However, $\mathrm{VO}_{2}$ peak correlated strongly with peak exercise CI, iLAVmin, LAEmF, LA reservoir, RAEmF and
RA reservoir but not with RVEF (Table 3). On multivariate analysis, peak exercise RAEmF and iLAVmin were independent predictors of $\mathrm{VO}_{2}$ peak $(B=0.58$ and $\beta=-0.52$, respectively) in CTEPH patients and together explained $72 \%$ of the variance in $\mathrm{VO}_{2}$ peak.

\section{Discussion}

In this study, we provide a comprehensive description of atrial physiology during exercise in healthy non-athletes, endurance athletes, and patients with chronic RV pressure overload due to CTEPH.

Normal physiology is characterized by an increase in atrial EF, whereas in CTEPH patients there is exerciseinduced dilation and dysfunction of the RA but also of the LA. Within the CTEPH cohort, measures of atrial volumes and function at peak exercise were independently associated with $\mathrm{VO}_{2}$ peak, whereas resting measures were not.

\section{Normal atrial physiology during exercise}

During exercise, cardiac output has to increase despite a reduction in filling time due to higher heart rate. Thus it seems logical that an increase in reservoir function plays an important role in accelerating LV filling by helping to maintain an enhanced atrioventricular pressure gradient

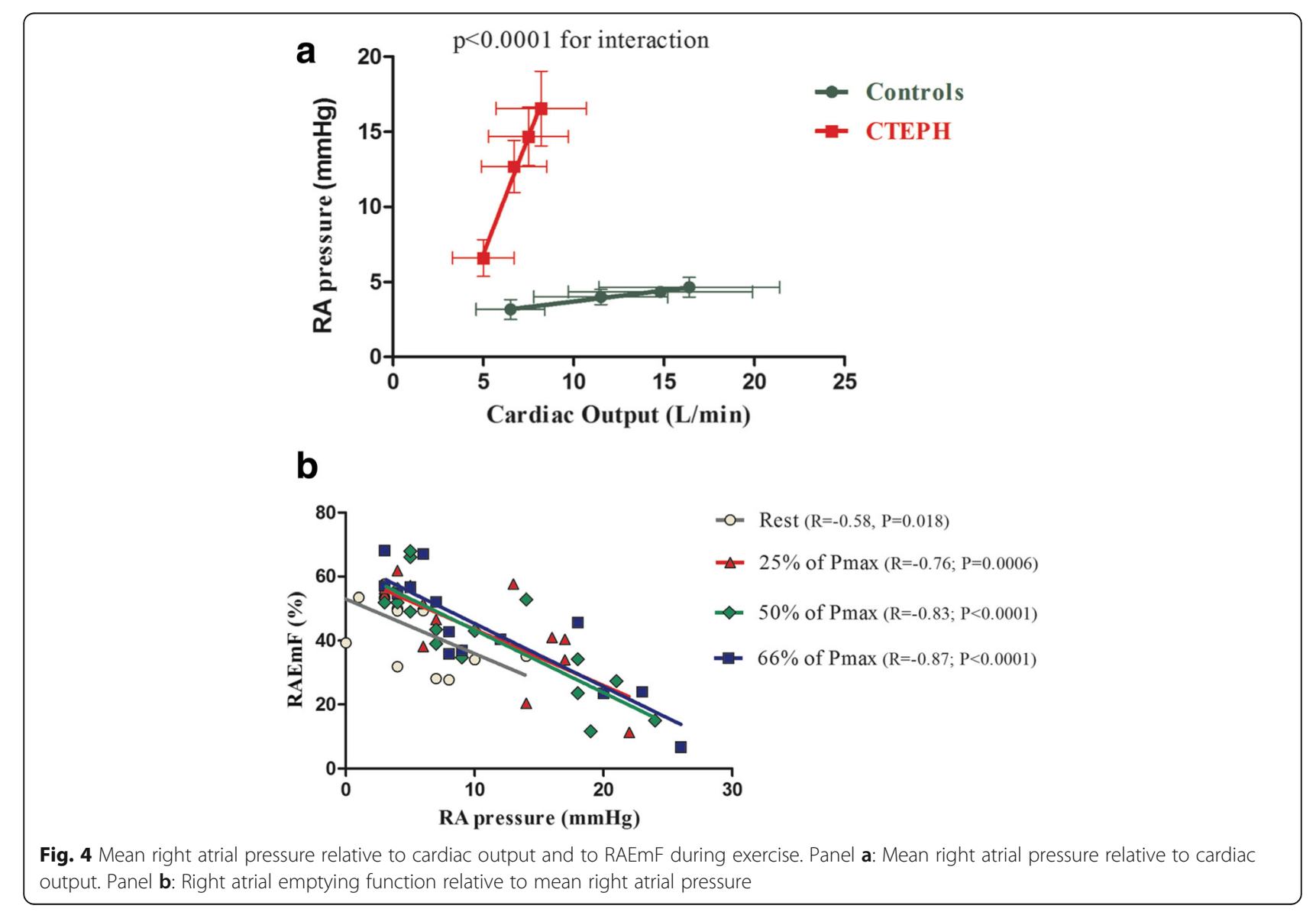


Table 3 Linear regression with $\mathrm{VO}_{2}$ peak $(\mathrm{ml} / \mathrm{min} / \mathrm{kg})$ in $\mathrm{CTEPH}$ patients

\begin{tabular}{|c|c|c|c|c|c|}
\hline \multirow[b]{2}{*}{ Parameter } & & \multicolumn{2}{|c|}{ Univariate analysis } & \multicolumn{2}{|c|}{ Multivariate analysis } \\
\hline & & $R$ & $P$-value & Beta & $P$-value \\
\hline Age & & -0.447 & 0.109 & & \\
\hline LVEDVi $\left(\mathrm{ml} / \mathrm{m}^{2}\right)$ & $\begin{array}{l}\text { Rest } \\
\text { Peak Ex }\end{array}$ & $\begin{array}{l}0.395 \\
0.464\end{array}$ & $\begin{array}{l}0.162 \\
0.110\end{array}$ & & \\
\hline LVESVi $\left(\mathrm{ml} / \mathrm{m}^{2}\right)$ & $\begin{array}{l}\text { Rest } \\
\text { Peak Ex }\end{array}$ & $\begin{array}{l}0.435 \\
0.328\end{array}$ & $\begin{array}{l}0.120 \\
0.273\end{array}$ & & \\
\hline LVEF (\%) & $\begin{array}{l}\text { Rest } \\
\text { Peak Ex }\end{array}$ & $\begin{array}{l}-0.305 \\
-0.072\end{array}$ & $\begin{array}{l}0.289 \\
0.816\end{array}$ & & \\
\hline RVEDVi $\left(m \mathrm{~m} / \mathrm{m}^{2}\right)$ & $\begin{array}{l}\text { Rest } \\
\text { Peak Ex }\end{array}$ & $\begin{array}{l}-0.192 \\
0.047\end{array}$ & $\begin{array}{l}0.510 \\
0.880\end{array}$ & & \\
\hline RVESVi $\left(\mathrm{ml} / \mathrm{m}^{2}\right)$ & $\begin{array}{l}\text { Rest } \\
\text { Peak Ex }\end{array}$ & $\begin{array}{l}-0.193 \\
-0.217\end{array}$ & $\begin{array}{l}0.509 \\
0.477\end{array}$ & & \\
\hline RVEF (\%) & $\begin{array}{l}\text { Rest } \\
\text { Peak Ex }\end{array}$ & $\begin{array}{l}0.046 \\
0.406\end{array}$ & $\begin{array}{l}0.876 \\
0.168\end{array}$ & & \\
\hline $\begin{array}{l}\text { Cardiac Index } \\
\left(1 / \mathrm{m}^{2}\right)\end{array}$ & $\begin{array}{l}\text { Rest } \\
\text { Peak Ex }\end{array}$ & $\begin{array}{l}0.043 \\
0.620\end{array}$ & $\begin{array}{l}0.885 \\
0.024\end{array}$ & & \\
\hline iLAVmax (ml/m²) & $\begin{array}{l}\text { Rest } \\
\text { Peak Ex }\end{array}$ & $\begin{array}{l}-0.135 \\
0.056\end{array}$ & $\begin{array}{l}0.646 \\
0.862\end{array}$ & & \\
\hline iLAVmin $\left(\mathrm{ml} / \mathrm{m}^{2}\right)$ & $\begin{array}{l}\text { Rest } \\
\text { Peak Ex }\end{array}$ & $\begin{array}{l}-0.116 \\
-0.631\end{array}$ & $\begin{array}{l}0.692 \\
0.028\end{array}$ & -0.515 & 0.018 \\
\hline LAEmF (\%) & $\begin{array}{l}\text { Rest } \\
\text { Peak Ex }\end{array}$ & $\begin{array}{l}-0.045 \\
0.640\end{array}$ & $\begin{array}{l}0.879 \\
0.025\end{array}$ & & \\
\hline LA reservoir & $\begin{array}{l}\text { Rest } \\
\text { Peak Ex }\end{array}$ & $\begin{array}{l}-0.041 \\
0.637\end{array}$ & $\begin{array}{l}0.888 \\
0.026\end{array}$ & & \\
\hline iRAVmax $\left(\mathrm{ml} / \mathrm{m}^{2}\right)$ & $\begin{array}{l}\text { Rest } \\
\text { Peak Ex }\end{array}$ & $\begin{array}{l}-0.436 \\
-0.170\end{array}$ & $\begin{array}{l}0.119 \\
0.598\end{array}$ & & \\
\hline iRAVmin $\left(\mathrm{ml} / \mathrm{m}^{2}\right)$ & $\begin{array}{l}\text { Rest } \\
\text { Peak Ex }\end{array}$ & $\begin{array}{l}-0.410 \\
-0.377\end{array}$ & $\begin{array}{l}0.146 \\
0.227\end{array}$ & & \\
\hline RAEmF (\%) & $\begin{array}{l}\text { Rest } \\
\text { Peak Ex }\end{array}$ & $\begin{array}{l}0.268 \\
0.684\end{array}$ & $\begin{array}{l}0.354 \\
0.014\end{array}$ & 0.581 & 0.003 \\
\hline RA reservoir & $\begin{array}{l}\text { Rest } \\
\text { Peak Ex }\end{array}$ & $\begin{array}{l}0.264 \\
0.643\end{array}$ & $\begin{array}{l}0.361 \\
0.024\end{array}$ & & \\
\hline
\end{tabular}

Peak Ex peak exercise, LVEDVi, RVEDVi left and right ventricular end diastolic volume index, LVESVi, RVEVSi left and right ventricular end systolic volume index, $L V E F, R V E F$ left and right ventricular ejection fraction, iLAVmax, iRAVmax indexed left and right maximal volume, iLAVmin, iRAVmin indexed left and right minimal volume, $L A E m F, R A E m F$ left and right atrial emptying function, $L A$ and $R A$ reservoir $L A$ and $R A$ reservoir function

during diastole and also by increasing LA booster function to increase ventricular preload. These theoretical presumptions are derived from resting data [1] and our data provides direct validation during exercise. Here we demonstrated that the physiological response to exercise is a decrease in atrial minimal volumes and an increase in atrial emptying function with exercise. These results are consistent with a previous echocardiographic study in athletes [4]. Although athletes had higher atrial volumes than non-athletes, exercise-induced atrial adaptations were similar in both groups. This observation confirms that atrial dilation can be considered to be part of the physiological remodeling to long-term endurance exercise, i.e. athlete's heart [17].
Abnormalities in atrial physiology are more apparent during exercise than at rest

Although pulmonary hypertension patients are often relatively asymptomatic at rest, their exercise capacity is severely reduced and symptoms typically occur with exertion. Recent studies in patients with pulmonary hypertension and CTEPH using cardiopulmonary exercise testing or RV imaging have demonstrated that exercise parameters relate better to prognosis and provide more insight into the mechanisms of exercise intolerance than resting parameters [13, 14, 21, 22]. Whilst several studies have focused on ventricular pathophysiology during exercise in CTEPH patients, less attention has been given to exerciseinduced changes in atrial volumes and function. The latter may be important, however, since resting mean RA pressure and iRAVmax are known strong predictors of outcome in patients with pulmonary hypertension [9-11].

RA pressure will only start to rise when RV enddiastolic pressure increases and is therefore considered a measure of RV failure [23]. iRAVmax represents an alternative, non-invasive marker of the severity of RV dysfunction. However, iRAVmax is not only determined by RV dysfunction and increases in mean RA pressure, but is also influenced by other factors such as endurance training [24]. At rest, we found that iRAVmax was even smaller in CTEPH patients than in athletes, despite marked differences between these groups in terms of RV and pulmonary vascular function. The impaired RV functional reserve in CTEPH patients, reflected by the lack of RVEF augmentation, was associated with increases in iRAVmax and iRAVmin from rest to peak exercise in CTEPH patients, contrasting with decreases in RA volumes in healthy non-athletes and athletes. As a result, RAEmF was diminished during exercise in CTEPH patients relative to healthy subjects. Thus, the changes in RA volumes during exercise enabled a far better distinction between physiological and pathological RA dilation than resting measures. Furthermore, atrial function may be less prone to confounders than atrial volume measures. Indeed, even at rest, RAEmF was reduced in CTEPH patients, albeit to a minor extent, whereas RAEmF was similar in athletes and non-athletes. In addition, we found that RAEmF correlated highly with mean RA pressure, both at rest and peak exercise, suggesting that it may be used as a noninvasive surrogate to assess RA pressure overload. On multivariate analysis, peak exercise RAEmF and iLAVmin were independent predictors of $\mathrm{VO}_{2}$ peak and better explained exercise limitation in CTEPH patients than direct measures of RV function such as RVEF or cardiac index.

\section{Atrio-ventricular interaction}

It is intriguing to speculate that RA function may be a more sensitive indicator of RV failure and pulmonary vascular disease than direct RV measures. The atria are 
thin-walled, compliant chambers with a tendency to dilate in response to chronic elevations in ventricular filling pressures. One hypothesis supported by invasive data $[25,26]$ contends that RV diastolic dysfunction precedes evidence of systolic impairment. As a result, changes in RA volumes and RA function may be anticipated and may precede evidence of RV systolic measures. This is analogous to the left-sided heart chambers where LA dilation and dysfunction is an early sign of LV diastolic dysfunction and is independently associated with survival [3]. Indeed, there is interplay among atrial function and ventricular performance throughout the cardiac cycle. Reservoir function is influenced by the descent of the ventricular base during systole, and conduit function is closely related to ventricular relaxation and compliance [20]. There was a discrepancy between atrial and ventricular stroke volume index. Indeed, atrial stroke volume is a misnomer, it is only a part of the total volume which actually goes through the atria, there is no method that is able to quantify this volume. Actually, the difference between LV and LA stroke volume defines atrial conduit volume $[27,28]$.

\section{Left atrial dysfunction as a sign of ventricular interdependence during exercise?}

Another interesting finding of this study is that functional abnormalities were not limited to the RA, but also affected the LA. Indeed, CTEPH patients had reduced LA function at rest and exercise; and iLAVmin decrease during exercise was impaired in comparison to non-athletes and athletes. This blunted LA functional response to exercise without an increase in LA volumes may be explained by an interdependence between the right and the left side of the heart whereby impaired RV contractile and stroke volume reserve during exercise results in progressive underfilling of the left heart, thereby decreasing both LA and LV preload. This interdependence was emphasized by the finding that LAEmF correlated with RVEF but not with LVEF. Furthermore, increases in iRAVmax and RVEDV result in septal shift which leads to compression and reduced filling of the LA and LV given the relatively non-distensible nature of the pericardium [29]. Hence, increasing RV filling pressures during exercise related to RV failure can secondarily alter LV filling pressures and geometry [23]. Thus, despite underfilling of the LA and LV, pulmonary capillary wedge pressure may have actually increased during exercise due to right heart overload and ventricular interaction. In keeping with this premise, Andersen et al. [30] elegantly demonstrated that patients with severe tricuspid regurgitation have a higher pulmonary capillary wedge pressure, which is entirely explained by a disproportionate increase in mean RA pressure whereas LV transmural pressure, i.e. effective LV distending pressure, drops during exercise. Similarly, inadequate diastolic filling together with increased pulmonary capillary wedge pressures may have explained the dysfunctional response of the LA during exercise in the CTEPH population in our study. This finding is of potential clinical relevance given our finding that peak exercise iLAVmin was independently associated with $\mathrm{VO}_{2}$ peak, the strongest predictor of outcome in patients with pulmonary hypertension $[13,14]$.

\section{Limitations and perspectives}

Firstly, given the constraints of recruiting healthy subjects for an invasive study protocol, we did not attempt to match the whole population of healthy nonathletes, athletes and CTEPH patients for age. Nevertheless, the small subgroup comparison with subjects of similar ages demonstrated similar RA changes to those in the global study population, and it has been previously demonstrated that the decrease in RAEmF with age is only mild [31].

Secondly, pulmonary capillary wedge pressure was only measured at rest in the CTEPH patients, whilst no wedge pressure measurements were obtained in controls due to the serious potential for serious adverse events in performing these measurements during exercise. Therefore, we could not assess the degree to which pulmonary capillary wedge pressure influenced the changes in iLAVmin and LAEmF observed during exercise.

Lastly, we only assessed global atrial emptying function and reservoir function [20] but not conduit and booster function, as this is currently not feasible given the temporal constraints of exercise imaging. Nevertheless, previous studies on LA function have demonstrated that global emptying function is the best predictor of elevated pulmonary capillary wedge pressure [6] and a clinical relevant predictor of outcome in heart failure [3].

It might be valuable to study more in details the arterioventricular interaction occurring in CTEPH patients by assessing pulmonary arteria stiffness during exercise CMR [32]. Also, the validation of exercise CMR in other forms of pulmonary hypertension could contribute to widespread the use of this new technic in clinical practice.

\section{Conclusion}

Augmentation of RAEmF and LAEmF can be observed during exercise in healthy subjects. In contrast, patients with CTEPH have impaired LAEmF and RAEmF, which becomes more apparent during exercise than at rest. As compared with resting measures, exercise provides a better means of distinguishing between normal right-sided heart function and early right-sided failure due to pulmonary vascular disease. Peak exercise RAEmF correlates highly with RA pressure and is associated with exercise capacity. 


\section{Additional files}

Additional file 1: Figure S1. Intra and inter observer variability of atrial volumes. Linear regressions with intra-class correlation coefficients. (TIFF 6593 kb)

Additional file 2: Table S4. Patients characteristics (age matched subgroups analysis). (DOCX $59 \mathrm{~kb}$ )

Additional file 3: Table S5. CMR volumes and hemodynamics at rest and peak exercise (age matched subgroups analysis). (DOCX 129 kb)

\section{Abbreviations}

CMR: Cardiovascular magnetic resonance; CPET: Cardiopulmonary exercise testing; CTEPH: Chronic thromboembolic pulmonary hypertension; EDV: Enddiastolic volume; ESV: End-systolic volume; iLAVmax and min: Indexed maximal and minimal left atrial volume; iRAVmax and min: Indexed maximal and minimal right atrial volume; LA: Left atrium/left atrial; LAEmF: Left atrial emptying function; LV: Left ventricle/left ventricular; LVEF: Left ventricular ejection fraction; Pmax: Maximal power output; RA: Right atrium/right atrial; RAEmF: Right atrial emptying function; RV: Right ventricle/right ventricular; RVEF: Right ventricular ejection fraction; SAX: Short-axis

\section{Acknowledgments}

Not applicable.

\section{Funding}

This work was supported by a grant from the Fund for Scientific Research Flanders (FWO), Belgium. FS was supported by a grant of the French Federation of Cardiology (FFC); ALG was supported by a scholarship from the National Health and Medical Research Council (NHMRC) and National Heart Foundation (NHF) of Australia.

\section{Availability of data and materials}

The datasets used during the current study are available from the corresponding author on reasonable request.

\section{Authors' contributions}

FS performed the acquisition of the data, analyzed the data and wrote the manuscript. GC, ALG and HH contributed to study design, data acquisition, data analysis and manuscript preparation; PC provided the in-house-developed software program (RightVol, Leuven, Belgium) assisted with the study design and revised the manuscript. MD contributed to the study design, subject recruitment and data acquisition. JB and FC revised the manuscript. All authors read and approved the final manuscript.

\section{Ethics approval and consent to participate}

The study protocol conformed to the Declaration of Helsinki and was approved by the ethics committee of UZ Leuven ( $N^{\circ}$ B322201214035). All subjects provided informed consent

\section{Consent for publication}

Not applicable.

\section{Competing interests}

The authors declare that they have no competing interests.

\section{Publisher's Note}

Springer Nature remains neutral with regard to jurisdictional claims in published maps and institutional affiliations.

\footnotetext{
Author details

${ }^{1}$ Department of Cardiology, University Hospital Gasthuisberg, University of Leuven, Leuven, Belgium. ²Department of Sport Medicine, University Hospital Pontchaillou, Rennes 1 University, Rennes, France. ${ }^{3}$ Baker IDI Heart and Diabetes Institute, Melbourne, Australia. ${ }^{4}$ Department of Cardiovascular Imaging and Dynamics, University of Leuven, Leuven, Belgium. ${ }^{5}$ Department of Imaging and Pathology, University Hospital Gasthuisberg, University of Leuven, Leuven, Belgium. ${ }^{6}$ Department of Pneumology, University Hospital Gasthuisberg, University of Leuven, Leuven, Belgium. ${ }^{7}$ Department of Cardiology, Antwerp University Hospital, University of Antwerp, Antwerp, Belgium.
}

Received: 2 August 2017 Accepted: 23 November 2017 Published online: 18 December 2017

\section{References}

1. Stefanadis C, Dernellis J, Toutouzas P. A clinical appraisal of left atrial function. Eur Heart J. 2001;22:22-36.

2. Vieira MJ, Teixeira R, Gonçalves L, Gersh BJ. Left atrial mechanics: echocardiographic assessment and clinical implications. J Am Soc Echocardiogr. 2014;27:463-78.

3. Pellicori $P$, Zhang J, Lukaschuk E, Joseph AC, Bourantas CV, Loh H, Bragadeesh T, Clark AL, Cleland JGF. Left atrial function measured by cardiac magnetic resonance imaging in patients with heart failure: clinical associations and prognostic value. Eur Heart J. 2015;36:733-42.

4. Gabrielli L, Bijnens BH, Brambila C, Duchateau N, Marin J, Sitges-Serra I, Mont L, Brugada J, Sitges M. Differential atrial performance at rest and exercise in athletes: potential trigger for developing atrial dysfunction? Scand J Med Sci Sports. 2016;26:1444-54.

5. Santoro A, Alvino F, Antonelli G, Molle R, Mondillo S. Left atrial strain after maximal exercise in competitive waterpolo players. Int J Cardiovasc Imaging. 2015;32:399-405.

6. Posina K, McLaughlin J, Rhee P, Li L, Cheng J, Schapiro W, Gulotta RJ, Berke $A D$, Petrossian GA, Reichek N, Cao JJ. Relation of phasic left atrial volume and emptying function to left ventricular filling pressure: a cardiovascular magnetic resonance study. J Cardiovasc Magn Reson. 2013;15:99.

7. Claessen G, Claus P, Ghysels S, Vermeersch P, Dymarkowski S, La Gerche A, Heidbuchel $\mathrm{H}$. Right ventricular fatigue developing during endurance exercise. Med Sci Sports Exerc. 2014;46:1717-26.

8. La Gerche A, Claessen G, Van De Bruaene A, Pattyn N, Van Cleemput J, Gewillig M, Bogaert J, Dymarkowski S, Claus P, Heidbuchel H. Cardiac magnetic resonance imaging: a new gold standard for ventricular volume quantification during high-intensity exercise. Circ Cardiovasc Imaging. 2012;6:329-38.

9. Zafrir N, Zingerman B, Solodky A, Ben-Dayan D, Sagie A, Sulkes J, Mats I, Kramer MR. Use of noninvasive tools in primary pulmonary hypertension to assess the correlation of right ventricular function with functional capacity and to predict outcome. Int J Cardiovasc Imaging. 2006;23:209-15.

10. Raymond RJ, Hinderliter AL, Willis PW, Ralph D, Caldwell EJ, Williams W, Ettinger NA, Hill NS, Summer WR, de Boisblanc B, Schwartz T, Koch G, Clayton LM, Jöbsis MM, Crow JW, Long W. Echocardiographic predictors of adverse outcomes in primary pulmonary hypertension. J Am Coll Cardiol. 2002;39:1214-9.

11. D'Alonzo GE, Barst RJ, Ayres SM, Bergofsky EH, Brundage BH, Detre KM, Fishman AP, Goldring RM, Groves BM, Kernis JT. Survival in patients with primary pulmonary hypertension. Results from a national prospective registry. Ann Intern Med. 1991;115:343-9.

12. Auger WR, Kerr KM, Kim NHS, Ben-Yehuda O, Knowlton KU, Fedullo PF. Chronic thromboembolic pulmonary hypertension. Cardiol Clin. 2004;22:453-66.

13. Grunig $E$, Tiede $H$, Enyimayew EO, Ehlken N, Seyfarth HJ, Bossone $E$, D'Andrea A, Naeije R, Olschewski H, Ulrich S, Nagel C, Halank M, Fischer C. Assessment and prognostic relevance of right ventricular contractile reserve in patients with severe pulmonary hypertension. Circ. 2013;128:2005-15.

14. Blumberg FC, Arzt M, Lange T, Schroll S, Pfeifer M, Wensel R. Impact of right ventricular reserve on exercise capacity and survival in patients with pulmonary hypertension. Eur J Heart Fail. 2014;15:771-5.

15. Claessen G, La Gerche A, Wielandts J-Y, Bogaert J, Van Cleemput J, Wuyts W, et al. Exercise pathophysiology and sildenafil effects in chronic thromboembolic pulmonary hypertension. Heart. 2015;101:637-44.

16. Claessen G, La Gerche A, Dymarkowski S, Claus P, Delcroix M, Heidbuchel H. Pulmonary vascular and right ventricular reserve in patients with normalized resting hemodynamics after pulmonary endarterectomy. J Am Heart Assoc. 2015:4:e001602.

17. Prior DL, La Gerche A. The athlete's heart. Heart. 2012;98:947-55.

18. Kim NH, Delcroix M, Jenkins DP, Channick R, Dartevelle P, Jansa P, Lang I, Madani MM, Ogino H, Pengo V, Mayer E. Chronic thromboembolic pulmonary hypertension. J Am Coll Cardiol. 2013;62:D92-9.

19. Lewis GD, Bossone E, Naeije R, Grünig E, Saggar R, Lancellotti P, Ghio S, Varga J, Rajagopalan S, Oudiz R, Rubenfire M. Pulmonary vascular hemodynamic response to exercise in cardiopulmonary diseases. Circ. 2013;128:1470-9.

20. Hoit B. Left atrial size and function. J Am Coll Cardiol. 2014;63:493-505.

21. Held M, Grün M, Holl R, Hübner G, Kaiser R, Karl S, Kolb M, Schäfers HJ, Wilkens $\mathrm{H}$, Jany B. Cardiopulmonary exercise testing to detect chronic 
thromboembolic pulmonary hypertension in patients with normal echocardiography. Respiration. 2014;87:379-87.

22. La Gerche A, Claessen G, Burns AT. To assess exertional breathlessness you must exert the breathless. Eur J Heart Fail. 2014;15:713-4.

23. Bemis CE, Serur JR, Borkenhagen D, Sonnenblick EH, Urschel CW. Influence of right ventricular filling pressure on left ventricular pressure and dimension. Circ Res. 1974;34:498-504.

24. D'Andrea A, Riegler L, Cocchia R, Scarafile R, Salerno G, Gravino R, Golia E, Vriz O, Citro R, Limongelli G, Calabrò P, Di Salvo G, Caso P, Russo MG, Bossone E, Calabrò R. Left atrial volume index in highly trained athletes. Am Heart J. 2010;159:1155-61.

25. Champion HC, Michelakis ED, Hassoun PM. Comprehensive invasive and noninvasive approach to the right ventricle-pulmonary circulation unit: state of the art and clinical and research implications. Circ. 2009;120:992-1007.

26. Murch SD, La Gerche A, Roberts T, Prior DL, Al MI, Burns AT. Abnormal right ventricular relaxation in pulmonary hypertension. Pulm Circ. 2015;5:370-5.

27. Leischik R, Littwitz H, Dworrak B, Garg P, Zhu M, Sahn DJ, et al. Echocardiographic evaluation of left atrial mechanics: function, history, novel techniques, advantages, and pitfalls. BioMed Research Int. 2015;2015:765921.

28. Bang CN, Dalsgaard M, Greve AM, Kober L, Gohlke-Baerwolf C, Ray S, et al. Left atrial size and function as predictors of new-onset of atrial fibrillation in patients with asymptomatic aortic stenosis: the simvastatine and ezetimibe in aortic stenosis study. Int J Cardiol. 2013;168:2322-7.

29. Marston NA, Auger WR, Madani MM, Kimura BJ, Strachan GM, Raisinghani $A B$, DeMaria AN, Blanchard DG. Assessment of left atrial volume before and after pulmonary thromboendarterectomy in chronic thromboembolic pulmonary hypertension. Cardiovasc Ultrasound. 2014;12:32.

30. Andersen MJ, Nishimura RA, Borlaug BA. The hemodynamic basis of exercise intolerance in tricuspid regurgitation. Circ Heart Fail. 2014;7:911-7.

31. Peluso D, Badano LP, Muraru D, Dal Bianco L, Cucchini U, Kocabay G, Kovacs A, Casablanca S, lliceto S. Right atrial size and function assessed with threedimensional and speckle-tracking echocardiography in 200 healthy volunteers. Eur Heart J Cardiovasc Imaging. 2013;14:1106-14.

32. Forouzan O, Warczytowa J, Wieben O, François CJ, Chesler NC. Non-invasive measurement using cardiovascular magnetic resonance of changes in pulmonary artery stiffness with exercise. J Cardiovasc Magn Reson. 2015;17:109.

\section{Submit your next manuscript to BioMed Central and we will help you at every step:}

- We accept pre-submission inquiries

- Our selector tool helps you to find the most relevant journal

- We provide round the clock customer support

- Convenient online submission

- Thorough peer review

- Inclusion in PubMed and all major indexing services

- Maximum visibility for your research

Submit your manuscript at www.biomedcentral.com/submit

) Biomed Central 